\title{
The preventative effect of bone marrow-derived mesenchymal stem cell exosomes on urethral stricture in rats
}

\author{
Zhengzhou Shi, Qi Wang, Dapeng Jiang \\ Department of Urology, Shanghai Children's Medical Center, Shanghai Jiao Tong University School of Medicine, Shanghai, China \\ Contributions: (I) Conception and design: All authors; (II) Administrative support: D Jiang; (III) Provision of study materials: Z Shi, Q Wang; (IV) \\ Collection and assembly of data: Z Shi, Q Wang; (V) Data analysis and interpretation: All authors. (VI) Manuscript writing: All authors; (VII) Final \\ approval of manuscript: All authors. \\ Correspondence to: Dapeng Jiang, PhD. Department of Urology, Shanghai Children's Medical Center, Shanghai Jiao Tong University School of \\ Medicine, 1678 Dongfang Road, Shanghai 200127, China. Email: jdp509@163.com.
}

\begin{abstract}
Background: Urethral stricture (US) is a major challenge in urology and there is an urgent need for effective therapies for its treatment. Exosomes derived from bone marrow mesenchymal stem cells (BMSCsExos) have been shown to be effective in preventing scar and fibrosis formation after tissue injury. However, the potential utility of BMSCs-Exos in the prevention of US remains unknown. We hypothesized that local administration of BMSCs-Exos may influence urethral healing and scar formation in a rat model of US.

Methods: A previously established model of rat US was used in this study. Sprague Dawley rats were randomly assigned into sham, US, and US + BMSCs-Exos groups. Micro-ultrasound assessment, histopathology, immunohistochemistry, and gene expression analysis were performed at four weeks postsurgery.

Results: US rats exhibited thick urethral walls with a narrowed lumen, when compared with sham rats. However, these changes were suppressed in the US + BMSCs-Exos group. The preventative effects of BMSCs-Exos on US formation were also apparent histologically. US + BMSCs-Exos rats demonstrated decreased expression of several fibrosis-related genes in urethral tissues, including Col I, fibronectin, and elastin, when compared with US rats. BMSCs-Exos treatment also led to an increase in the expression of angiogenesis-related genes in these tissues, including VEGF, eNOS, and bFGF.

Conclusions: Our findings therefore demonstrate that the local administration of BMSCs-Exos prevents urethral stricture formation by regulating fibrosis and angiogenesis. These findings provide a basis for an innovative strategy involving the clinical application of exosomes to counteract US formation.
\end{abstract}

Keywords: Urethral stricture (US); mesenchymal stem cell (MSC); exosome; fibrosis; healing

Submitted Mar 28, 2020. Accepted for publication Sep 13, 2020.

doi: $10.21037 /$ tau-20-833

View this article at: http://dx.doi.org/10.21037/tau-20-833

\section{Introduction}

Urethral stricture (US) is a narrowing of the urethra that is associated with fibrotic scarring. Effective treatment of US is challenging and, therefore, a subject of major concern in the field of urology. Various surgical options are available for the treatment of US, including urethral dilatation, internal urethrotomy, and urethroplasty (1-4). Antifibrotic drugs and growth factors have also been used in the treatment of US $(5,6)$. However, these surgical options and adjunctive treatments can result in complications and, furthermore, the stricture inevitably recurs. As such, current modalities for the treatment of US are ultimately of little therapeutic benefit.

Mesenchymal stem cells (MSCs) represent an attractive solution for the promotion of tissue repair and regeneration (7-9). In recent years, MSCs have been used to modulate fibrosis, apoptosis, inflammation, and ischemia at sites 
of injury, resulting in enhanced tissue healing $(10,11)$. Excessive fibrosis and reduced angiogenesis are associated with the pathogenesis of US. The potential for MSCs to suppress US by decreasing fibrosis has been documented extensively (12). However, the poor viability of MSCs after transplantation, possibly as a consequence of the unfavorable microenvironment of the repair site, limits their therapeutic potential in the treatment of this disease.

Many investigators have suggested that MSC exosomes can function as complex paracrine factors that mediate many of the functions of the cells from which they are derived (13-15). In particular, there is increasing evidence that exosomes derived from bone marrow mesenchymal stem cells (BMSCs) have potential utility in the treatment of tissue and organ fibrosis $(16,17)$. BMSCs-derived exosomes (BMCSs-Exos) play important roles in the regulation of a variety of biological processes. It has been shown that BMSCs-derived extracellular vesicles have a protective effect against ureteral injury-associated fibrosis in rats (18). It is therefore possible that BMCSs-Exos may have clinical utility in the treatment of US. In this study, we analyzed the antifibrotic effects of local injection of BMSCs-Exos on the quality of urethral tissue repair in a rat model of US.

We present the following article in accordance with the ARRIVE reporting checklist (available at http://dx. doi. org/10.21037/tau-20-833).

\section{Methods}

\section{Isolation and culture of rat BMSCs}

Eight Sprague Dawley (SD) rats were used for the isolation of BMSCs. Animals and procedures used in this study were approved by Animal Care and Use Committee at Shanghai Children's Medical Center, Shanghai Jiao Tong University School of Medicine (Shanghai, China). The document ID is SCMCIACUC-K2019025. BMSCs were isolated from rat bone marrow as described previously (19). Briefly, bone marrow was flushed from the tibias of rats and mononuclear cells then isolated from the bone marrow suspension by Ficoll density gradient centrifugation. The mononuclear cell pellets were resuspended in alpha-modified Eagle's medium containing $10 \%$ fetal bovine serum. The cells were then cultured in T-75 flasks at $37^{\circ} \mathrm{C}$ in a humidified atmosphere containing $5 \% \mathrm{CO}_{2}$. After $48 \mathrm{~h}$, the non-adherent cells were discarded and the adherent cells were further expanded until the culture was approximately $80 \%$ confluent. After three generations, the BMSCs were harvested and characterized.
All BMSCs used in this study were between passage 3 and 5 .

\section{Isolation and identification of BMSCs-Exos}

Once BMSCs cultures reached 80-90\% confluency, flasks were washed three times with PBS and the cells then cultured in Mesen Gro hMSC medium for an additional $48 \mathrm{~h}$. The resulting conditioned media was collected and exosomes then isolated as described previously (20). Briefly, the culture medium was subjected to a preclearing centrifugation step at $1,000 \times \mathrm{G}$ for $15 \mathrm{~min}$ to remove cellular debris, and then subjected to ultracentrifugation at $100,000 \times \mathrm{G}$ for $2 \mathrm{~h}$ to obtain a pellet containing the exosomes. The pellet was resuspended in $200 \mu \mathrm{L}$ of PBS and this exosome suspension was then subjected to vacuumassisted filtration using a $0.22-\mu \mathrm{m}$ PES filter. The filtered suspension was then centrifuged at $1,500 \times \mathrm{G}$ for $30 \mathrm{~min}$ using a $100-\mathrm{kDa}$ molecular weight cutoff hollow fiber membrane (Millipore, Billerica, MA, USA), and then passed once more through a $0.22-\mu \mathrm{m}$ PES filter. The total protein concentration of the exosome sample was determined using the Micro Bicinchoninic Acid Protein Assay Kit (Pierce). Exosomes were used immediately for downstream experiments. Transmission electron microscopy and western blotting were used to evaluate the quality of exosome samples.

\section{US model and treatment}

Forty-five male SD rats (weighing between 200 and $230 \mathrm{~g}$ ) were used in the US animal study. The rats were randomly divided into three groups, each consisting of 15 animals. These included a sham group, a US group, and a US + BMSCs-Exos group. The surgical procedures used in the rat US model were performed as described previously (12). On the day of surgery, a urethral support tube was administered to rats anesthetized with pentobarbital sodium. After administration of the indwelling urethral support tube, the ventral skin of the penis was cut to expose the urethra. Then, $100 \mu \mathrm{L}$ of saline containing $1 \mu \mathrm{g}$ human recombinant TGF- $\beta 1$ (PeproTech, NJ, USA) was injected locally into the urethral wall. Rats in the sham group received a salineonly injection to the urethra. After injection, the rats of the US and US + BMSCs-Exos groups received four partial incisions to the urethral wall with a $23 \mathrm{G}$ needle. The urethral support tube was then removed, and the skin of the penis sutured. BMSCs-Exos (200 $\mu \mathrm{g}$ in $100 \mu \mathrm{L}$ saline) or saline $(100 \mu \mathrm{L})$ control were then injected into the urethral 
Table 1 Rat primers used for qRT-PCR analysis.

\begin{tabular}{ll}
\hline Gene & Primer sequence \\
\hline COL1a1 & F: 5'- CCGGACTGTGAGGTTAGGAT -3' \\
R: 5'- AACCCAAAGGACCCAAATAC -3' \\
COL3a1 & F: 5'- AACGGAGCTCCTGGCCCCAT -3' \\
& R: 5'- ATTGCCTCGAGCACCTGCGG -3' \\
elastin & F: 5'- AAAACCCCCGAAGCCCTATG-3' \\
& R: 5'- TCACTTCTCTTCCGGCCAC -3' \\
fibronectin & F: 5'- GATTCTTCTGGCGTCTGCAC-3' \\
& R: 5'- GCCCCGGAACATGAGGATAG-3' \\
VEGF & F: 5'-GCAGAAGGAGGAGGGCAG-3' \\
& R: 5'- CACCAGGGTCTCGATTGGAT-3' \\
bFGF & F: 5'-CGCACCCTATCCCTTCACAGC-3' \\
& R: 5'- CAGCCTTCCACCCAAAGCAGT -3' \\
eNOS & F: 5'- TGACCCTCACCGATACAACA -3' \\
& R: 5'- CGGGTGTCTGATCCATGC -3' \\
GAPDH & F: 5'- TGACTCTACCCACGGCAAGTCAA-3' \\
& R: 5'- ACGACATACTCAGCACCAGCATCA-3'
\end{tabular}

bFGF, basic fibroblast growth factor; eNOS, endothelial nitric oxide synthase; VEGF, vascular endothelial growth factor; vWF, von Willebrand factor; GAPDH, glyceraldehyde-3-phosphate dehydrogenase.

wall on the following day. All rats underwent an ultrasound examination at 4 weeks after surgery. Penile and urethral tissues were harvested for histology, immunohistochemistry, and gene expression analysis after the animals were euthanized.

\section{Micro-ultrasound assessment}

Rats were anesthetized by isoflurane inhalation at 4 weeks following injury, and micro-ultrasound then performed to evaluate stricture of the urethra using a Vevo 2100 Imaging System (VisualSonics Inc. Canada). The procedure was repeated three times by the same examiner who was blinded to treatment allocation.

\section{Histology and immunobistochemistry}

Urethral specimens were harvested, fixed and further processed for histology and immunohistochemistry. Hematoxylin-eosin (H\&E) and Masson's trichrome staining were used to evaluate the effects of BMSCs-Exos on the degree of urethral stenosis and fibrosis. The urethral fibrosis score was evaluated as described previously (21).
Immunohistochemical staining with anti-rat von Willebrand Factor (vWF) antibody (Santa Cruz Biotechnology, CA) was performed to evaluate the structure of the epithelium.

\section{Gene expression}

Gene expression changes in urethral tissues were determined using real time PCR (RT-PCR). Total RNA from tissues was extracted using an RNeasy mini kit (Qiagen, Hilden, Germany). Total RNA isolation, cDNA synthesis, and gene expression assays were performed as described previously (22). Normalized gene expression data were calculated using the $2^{\Delta \mathrm{CT}}$ formula. Gene expression values were normalized to the reference gene glyceraldehyde-3phosphate dehydrogenase (GAPDH).

The expression of angiogenesis related genes encoding vascular endothelial growth factor (VEGF), endothelial nitric oxide synthase (eNOS), and basic fibroblast growth factor (bFGF), and fibrosis-associated genes encoding Col I, Col III, fibronectin, and elastin, were analyzed by RT-PCR. PCR primer sequences for the amplification of target genes are listed in Table 1.

\section{Statistical analysis}

All values are expressed as means \pm standard deviation. Group data were compared by analysis of variance (ANOVA) followed by Bonferroni's multiple comparison tests. Statistical analysis was performed using the SPSS 11.0 statistical package. $\mathrm{P}$ values $<0.05$ were considered statistically significant.

\section{Results}

\section{Characterization of BMSCs and BMSCs-Exos}

As shown in Figure 1A, the third generation BMSCs were spindle-shaped. In the flow cytometric analysis (Figure 1B), BMSCs exhibited high levels of markers CD44 and CD90 (66.6\% and $99.59 \%$ respectively), and very low levels of markers CD11b and CD34 (0.01\% and $3.51 \%$ respectively). Third generation BMSCs were successfully differentiated into adipogenic, osteogenic and chondrogenic cells (Figure 1C). As shown in Figure 2A, high levels of the characteristic marker proteins CD9, CD63 and HSP70 were detected by western blotting. Besides, the BMSCs-Exos were cup-shaped particles with a diameter of 50-150 $\mathrm{nm}$ (Figure $2 B$ and $C$ ). 
A

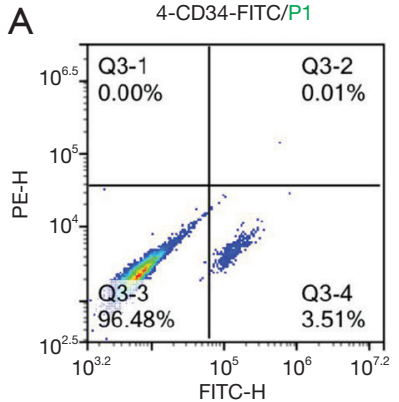

B

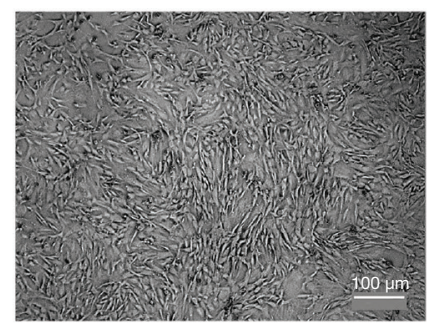

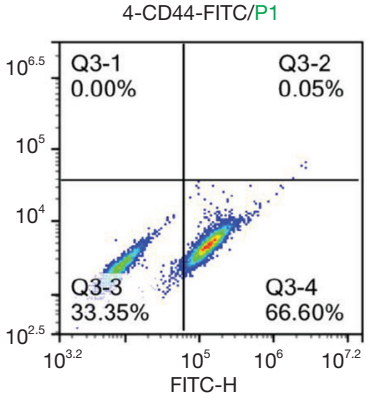
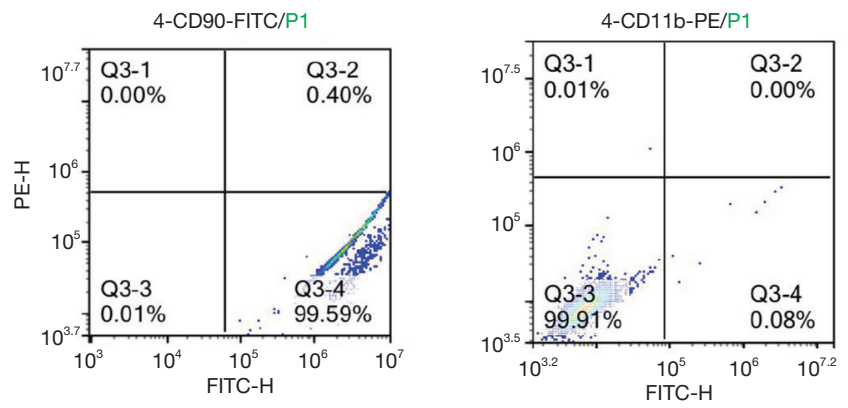

C
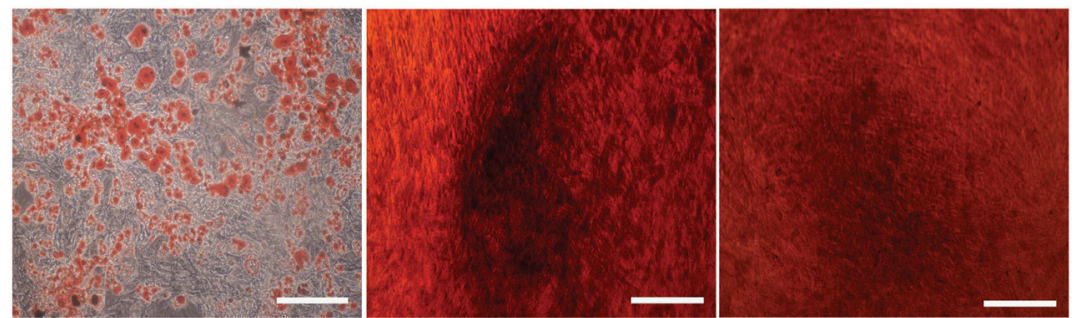

Figure 1 Characterization of BMSCs. (A) flow cytometric analysis of BMSCs surface markers. (B) morphology of BMSCs. (C) adipogenic, osteogenic, and chondrogenic differentiation potentials of BMSCs. Scale bar: $100 \mu \mathrm{m}$.

A

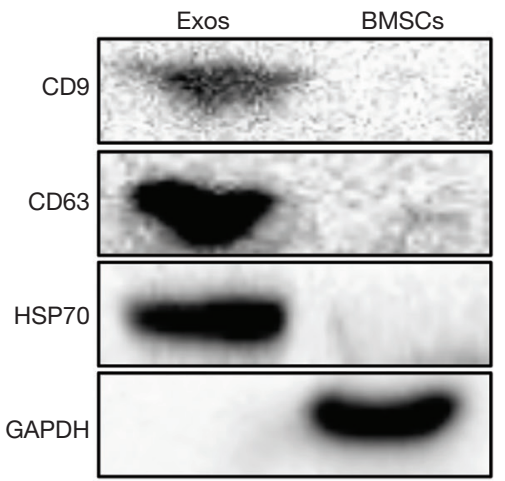

B

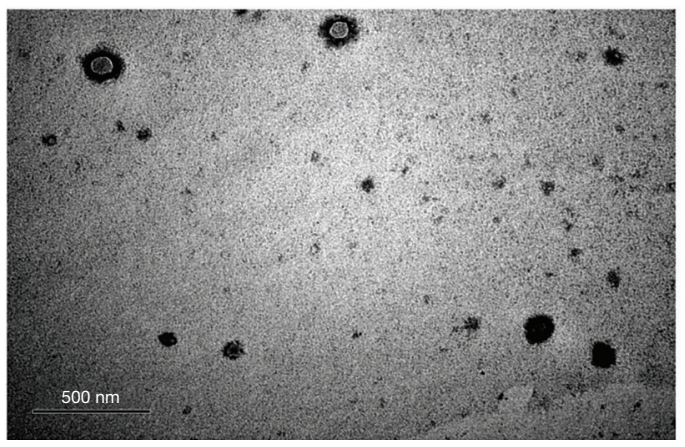

C

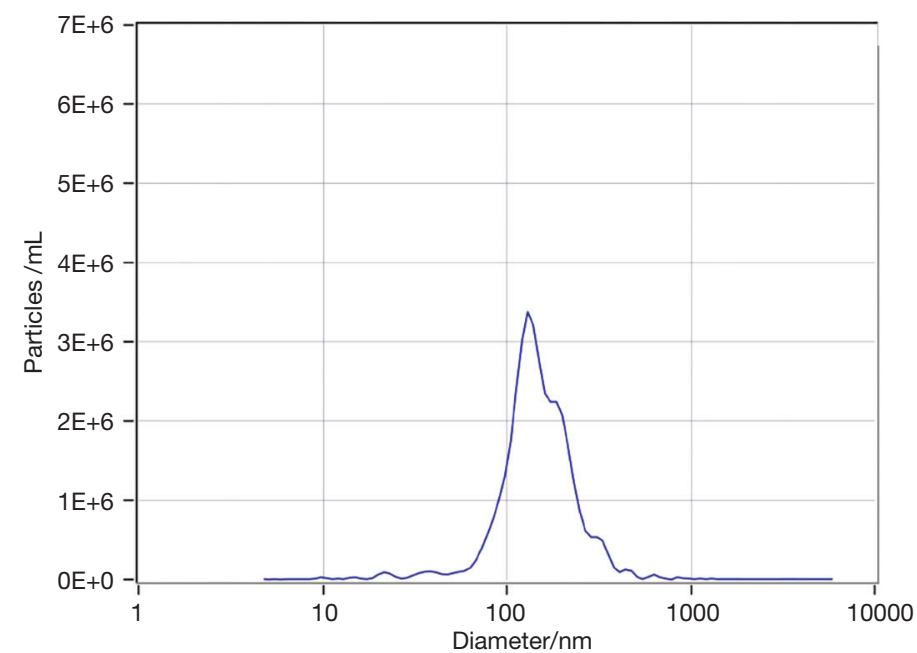

Figure 2 Characterization of BMSC-Exos. (A) western blotting was used to confirm the expression of BMSCs-Exos specific markers. (B) representative transmission electron microscopy image of BMSCs-Exos. (C) particle size distribution. Scale bar: $0.5 \mu \mathrm{m}$. 

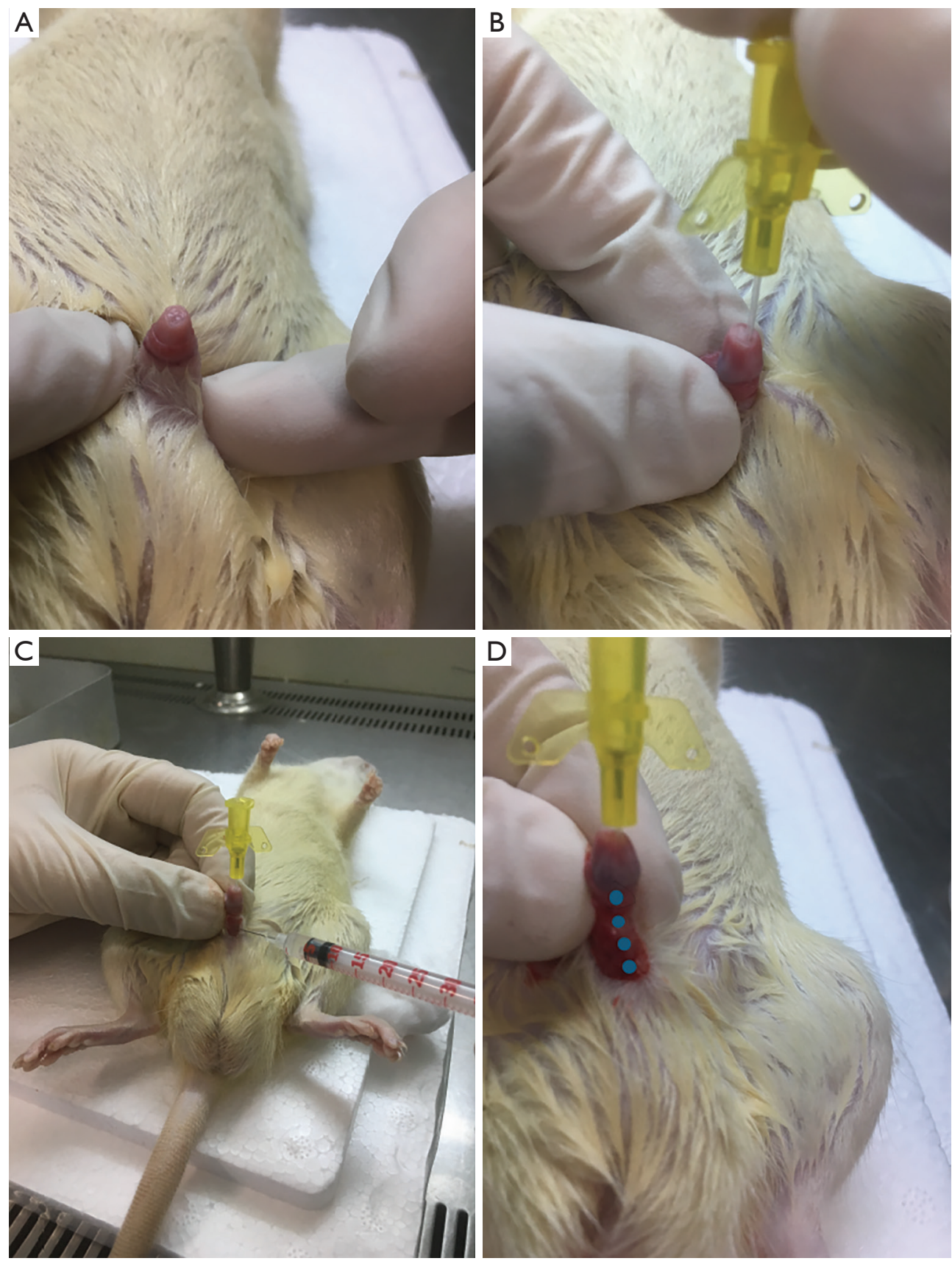

Figure 3 Rat US model. (A) penile of rat. (B) administration of the indwelling urethral support tube. (C) injection of human recombinant TGF- $\beta 1$. (D) site of penile injuries.

\section{Animals}

The local administration of TGF- $\beta 1$ (Figure 3) and BMSCsExos had no adverse effects on the overall well-being of rats. There were also no statistically significant differences in the weight of animals among the three treatment groups during the experimental period.

\section{Micro-ultrasound}

We conducted micro-ultrasound analysis 28 days after urethral injury to evaluate the effects of treatments on 

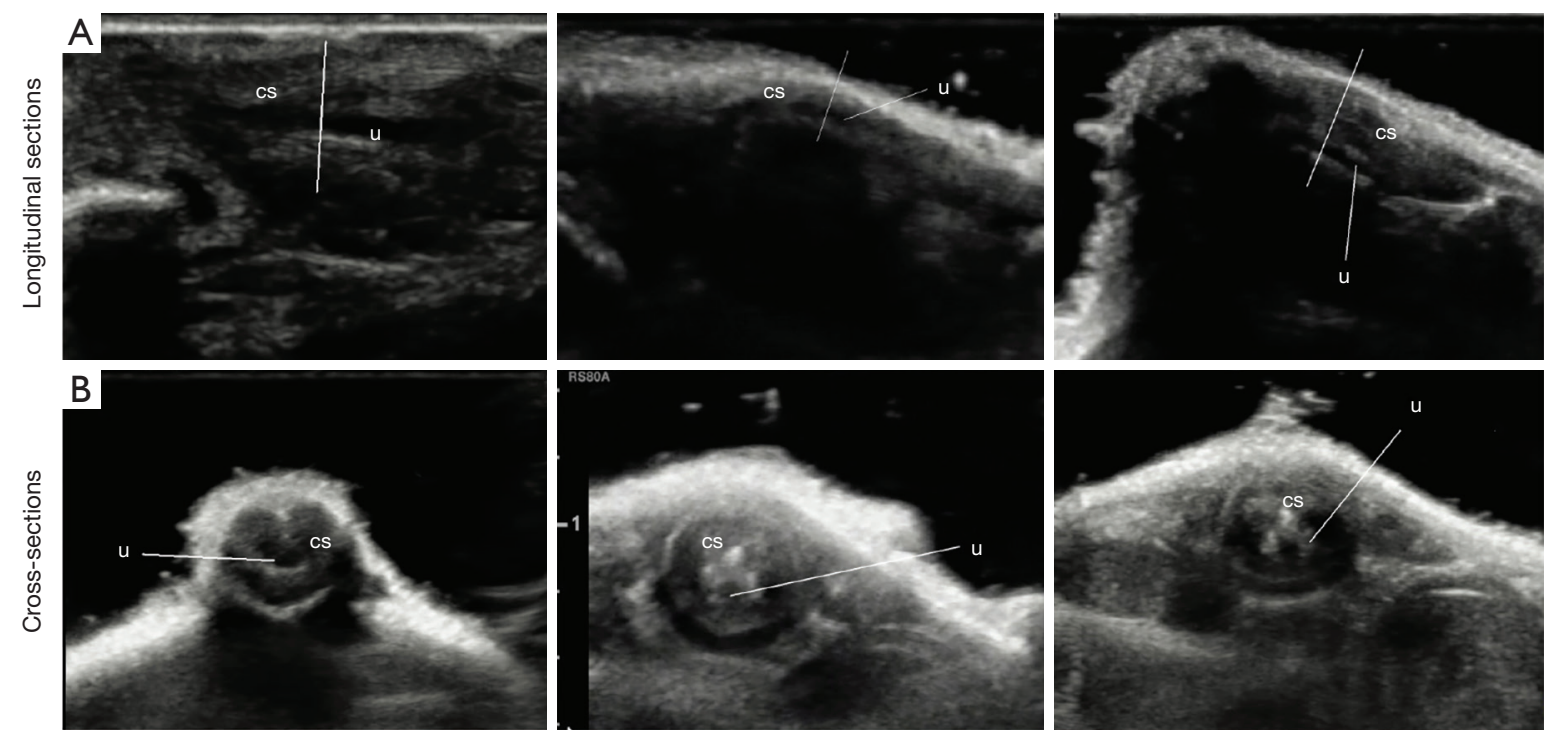

Sham

US

BMSCs-Exos

Figure 4 Representative micro-ultrasound images of injured penile urethras at 4 weeks after injury. (A) longitudinal sections. (B) crosssections. cs = corpus spongiosum; $\mathrm{u}=$ urethral lumen.

urethral morphology. As shown in Figure 4, microultrasound analysis revealed obvious urethral stricture in rats of the US group, while these changes were essentially absent in rats of the US + BMSCs-Exos group.

\section{Histological analysis}

Next, we investigated the effects of BMSCs-Exos on urethral stricture by H\&E and Masson's trichrome staining at 4 weeks post-implantation. Histological analysis of urethral tissue cross-sections revealed significant submucosal fibrosis with densely packed collagen fibers in US rats (Figure 5). However, there was only mild submucosal urethral fibrosis in rats treated with BMSCsExos. Immunohistological analysis of $\mathrm{vWF}$ staining suggested that the epithelium and the muscle layer were not fully formed in the US group. In the BMSCs-Exos group, the urethral mucosa showed an improved histological appearance with a well-formed epithelium (Figure 6).

\section{Gene expression}

Our RT-PCR analysis (Figure 7) also confirmed high levels of gene expression for fibrosis-related markers such as $\mathrm{Col}$ I, Col III, fibronectin, and elastin, in the penile midshaft tissues of rats in the US group. BMSCs-Exos treatment significantly decrease the expressions of these fibrosis- related genes, when compared with the US group $(\mathrm{P}<0.05)$. Next, we investigated the effect of BMSCs-Exos on the expression of angiogenesis-related genes, including VEGF, eNOS, and bFGF. Increased levels of eNOS and bFGF expression were detected in the penile midshaft tissues of BMSCs-Exos-treated rats, when compared with rats of the US and sham treatment groups $(\mathrm{P}<0.05)$.

\section{Discussion}

US is a fibrotic process associated with extensive collagen synthesis within the epithelium and surrounding tissues (23). Management of US is complex and represents a major concern in urology. Tissue fibrosis is the main etiopathological factor in urethral stricture development. Marked fibrosis following urethral injury has been identified as a key factor in US development (24). Antifibrotic medication therefore provides a potential therapeutic strategy for the treatment of US (5). Previous studies have found that BMSCs-Exos treatment improves the quality of tissue repair by inhibiting fibrosis and scar formation (25-27). However, the effects of BMSCsExos on the formation of US have not previously been elucidated.

In order to investigate the effect of BMSCs-Exos on urethral fibrosis, urethral tissues were collected for $\mathrm{H} \& \mathrm{E}$ and Masson's trichrome staining at 28 days-post tissue 

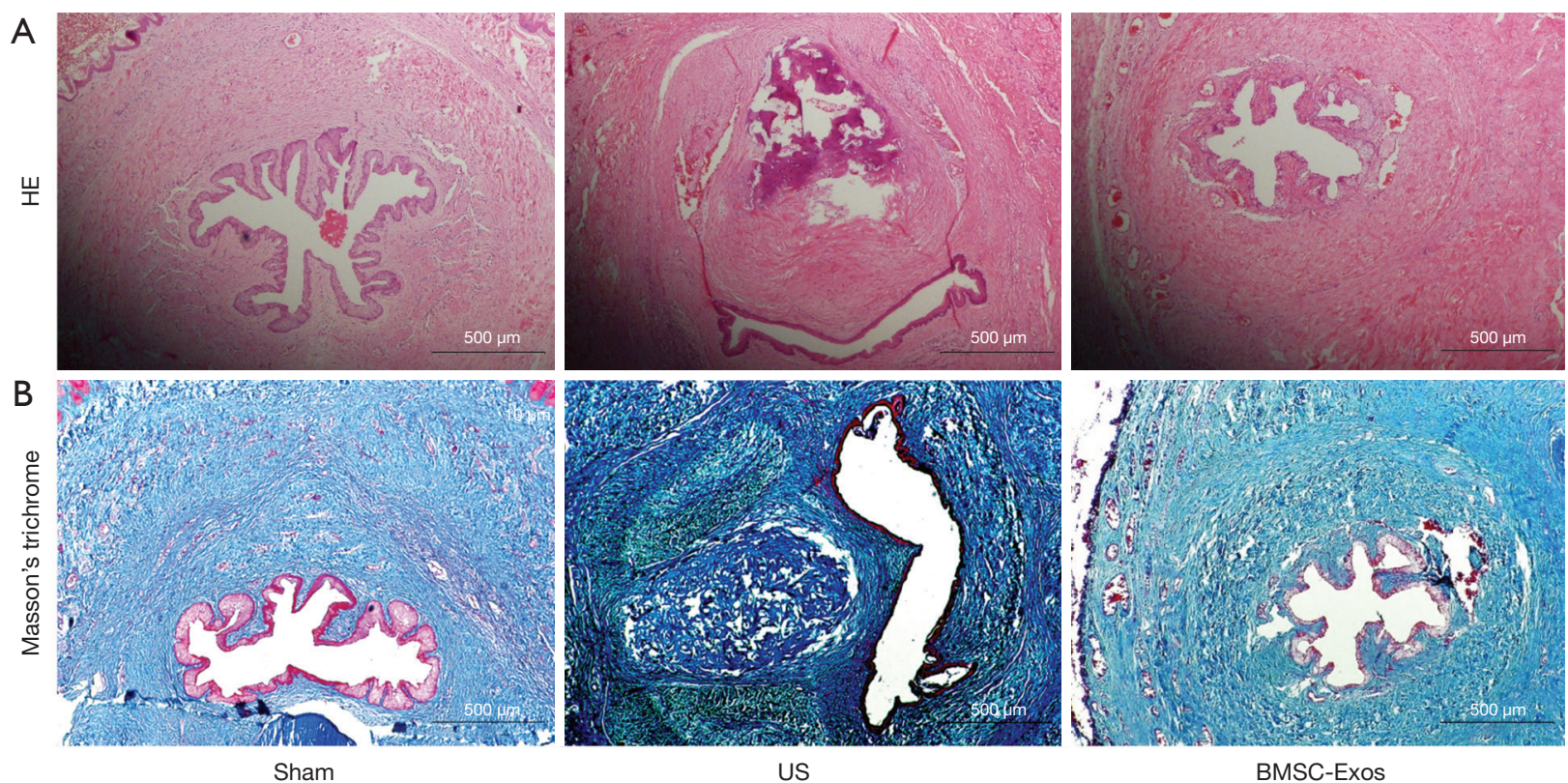

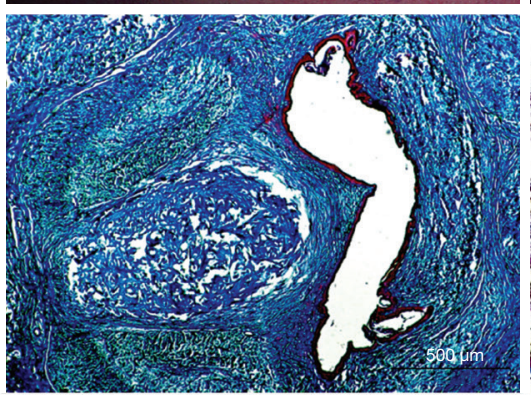

US

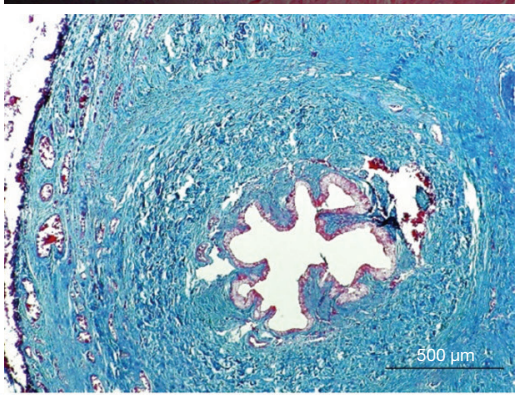

BMSC-Exos

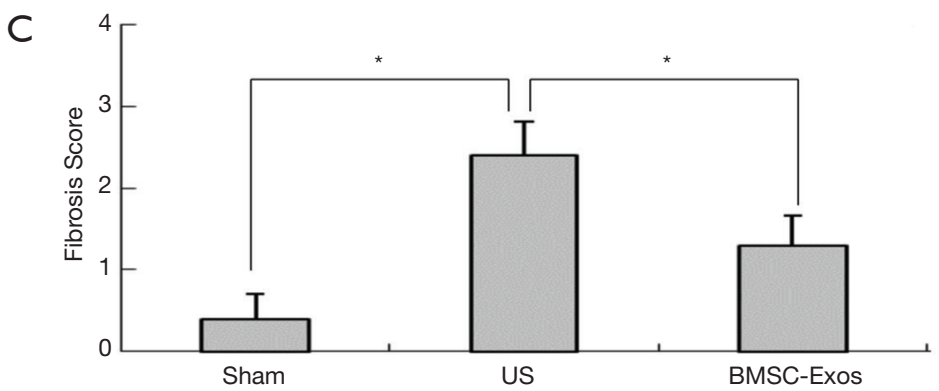

Figure 5 The results of histological evaluation for US at 4 weeks after injury. (A) The HE staining of urethral tissue cross-sections. (B) Masson's trichrome staining of urethral tissue cross-sections. (C) Fibrosis score was significantly lower for the rats in the BMSCs-Exos group compared with other groups ( $\mathrm{n}=8$ donors). Bars: $500 \mu \mathrm{m}$, magnification: $\times 10$. Data are represented as mean $\pm \mathrm{SD}$. ${ }^{*} \mathrm{P}<0.05$.

injury. Extensive collagen deposition was observed in the submucosal connective tissue of rats in both US groups. Disorganized collagen fibers were also evident in the healing urethra of US rats. Our micro-ultrasound analysis revealed varying degrees of bulbar urethral narrowing in US rats. However, the application of BMSCs-Exos to the site of urethral injury had a protective effect on rat US. Our H\&E and Masson's trichrome staining also demonstrated the antifibrotic properties of BMSCs-Exos in rat US. Moreover, our immunohistochemical analysis of $\mathrm{vWF}$ expression also suggested that administration of BMSCs-Exos improves the formation of the epithelium post-injury.

Ischemia is believed to contribute to the progression of urethral stricture through its inhibitory effects on tissue repair and promotion of excessive scar formation (28).
Several studies have showed that the exogenous application of BMSCs-Exos can enhance wound healing by inducing angiogenesis (29-31). In this study, we investigated the angiogenic potential of BMSCs-Exos in our rat model of US. Our experiments revealed that the local administration of BMSCs-Exos significantly increased the expression of angiogenic genes, such as eNOS, bFGF, and VEGF, in the healed urethral tissues of US rats. Previous studies have also shown that iNOS and eNOS can exert antioxidative and antifibrotic effects that promote improved tissue repair $(32,33)$. Our PCR results therefore suggest a role for NOS in mediating the antifibrotic properties of BMSCs-Exos towards US.

In this study, we successfully applied a rat model of US to examine the potential therapeutic effects of BMSCs-Exos. 


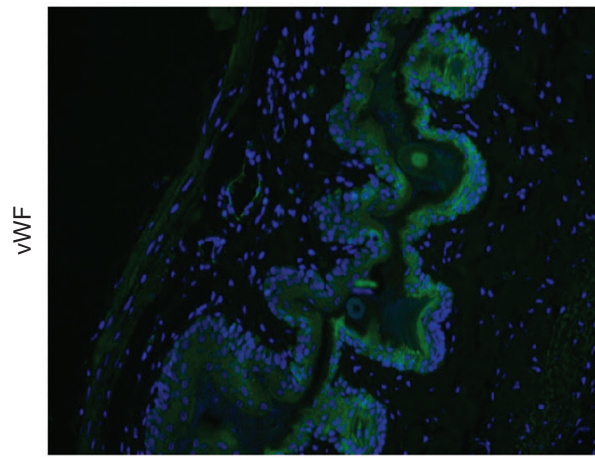

Sham

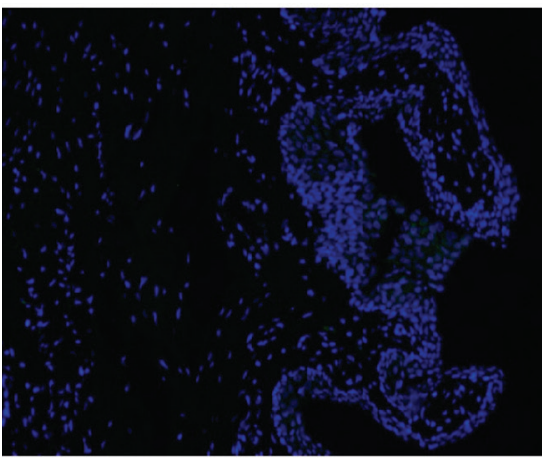

US

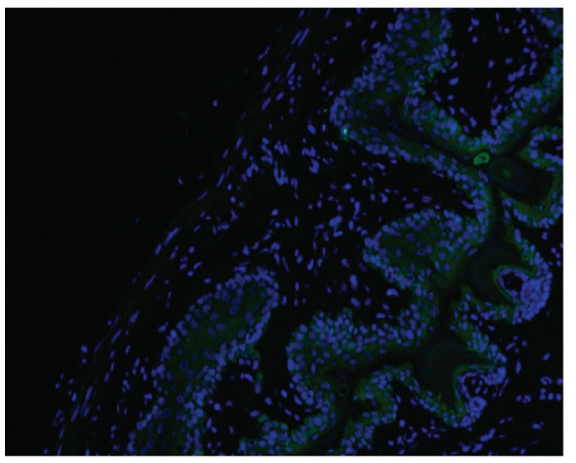

BMSCs-Exos

Figure $6 \mathrm{vWF}$ immunofluorescence staining results. The urethral mucosa showed an improved histological appearance with a well-formed epithelium In the BMSCs-Exos group. Magnification: $\times 40$.
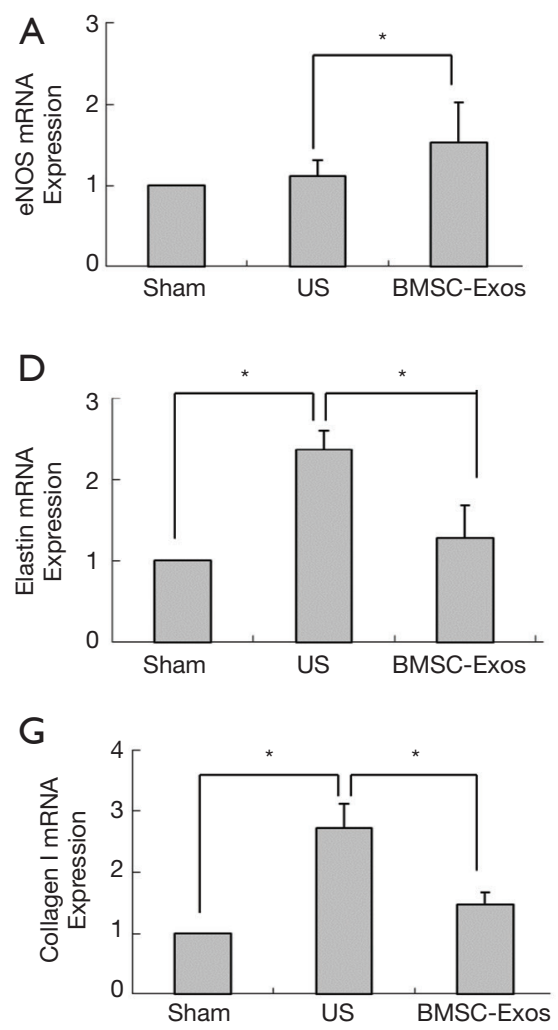
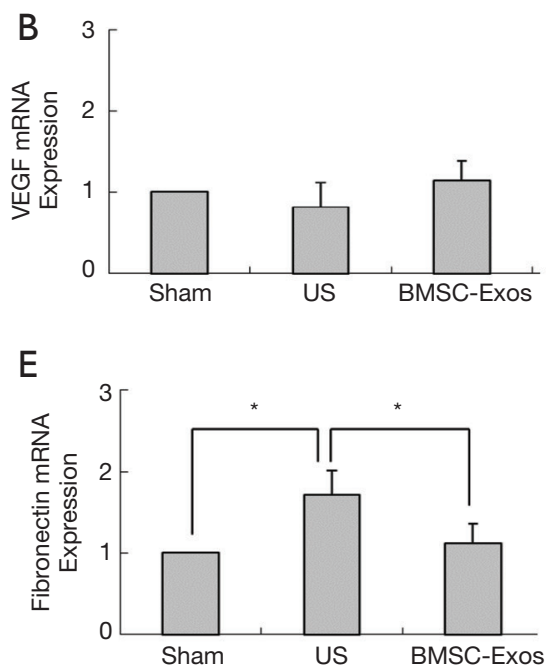
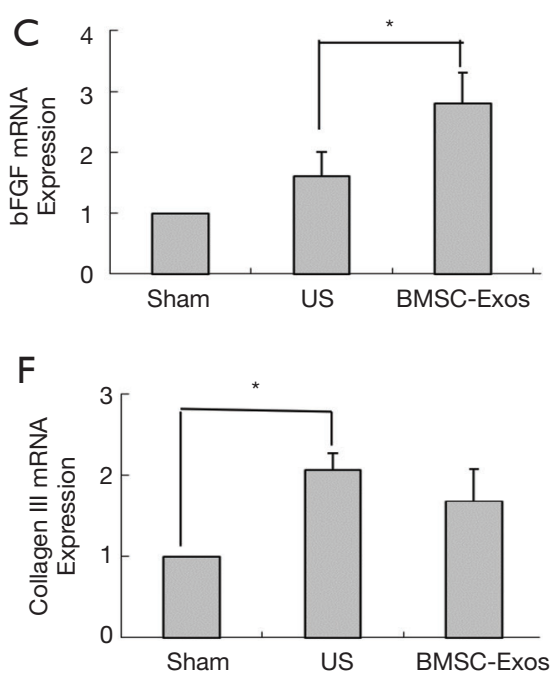

Figure 7 The expression of angiogenesis-related genes (eNOS, VEGF, and bFGF.) and fibrosis-related genes (Col I, Col III, fibronectin, and elastin) in the penile midshaft tissues of rats ( $\mathrm{n}=8$ donors). Data are represented as mean $\pm \mathrm{SD} .{ }^{*} \mathrm{P}<0.05$.

Our pathological examination confirmed the narrowing of the urethral lumen and excessive fibrotic scarring of the injured urethra that are hallmarks of US. Extracellular matrix and collagen deposition is a major characteristic of scar and fibrosis formation. In this study, we examined
mRNA expressions levels of Col I, Col III, fibronectin, and elastin, known regulators of fibrosis. Our RT-PCR analysis revealed that Col I, elastin, and fibronectin expression levels were significantly elevated in the penile midshaft tissues of rats in the US group. However, the local administration 
of BMSCs-Exos to injured urethral tissues inhibited the expression of these fibrosis-related genes, a finding that is consistent with those of our histological analysis.

It is important to highlight some limitations associated with the present study. Firstly, only one dose of BMSCsExos was used for the prevention of US. Future studies will need to evaluate the dose-dependent effects of BMSCsExos in the treatment of US before the potential translation of this therapy into the clinical setting. Secondly, the longterm effects of BMSCs-Exos on US were not assessed. Again, future studies focusing on the longer-term effects of this treatment strategy are warranted. A final limitation is that we have not address the molecular mechanisms responsible for mediating the suppressive effects of BMSCs-Exos towards US. A better understanding of the regulatory pathways by which BMSCs-Exos inhibit urethral fibrosis will facilitate the future development of therapeutic approaches for the effective prevention of US. However, in spite of these limitations, we believe that our study highlights the therapeutic potential of BMSCs-Exos in the clinical treatment of US.

In summary, we have found that BMSCs-Exos have a protective effect against fibrosis in a rat model of US. The ability of BMSCs-Exos to suppress fibrosis during urethral repair highlights the potential for the translation of this method into the clinical setting for the treatment of US.

\section{Acknowledgments}

Funding: This work was supported by grants from the Natural Science Foundation of Shanghai (grant number: 19ZR1432800), and the Program for improving the Clinical Scientific Research ability of Postgraduate students in Pediatrics, Shanghai Jiao Tong University School of Medicine (grant number: EKKY2018005DGD).

\section{Footnote}

Reporting Checklist: The authors have completed the ARRIVE reporting checklist. Available at http://dx. doi. org/10. 21037/tau-20-833

Data Sharing Statement: Available at http://dx. doi. org/10.21037/tau-20-833

Conflicts of Interest: All authors have completed the ICMJE uniform disclosure form (available at http://dx. doi. org/10.21037/tau-20-833). The authors have no conflicts of interest to declare.

Ethical Statement: The authors are accountable for all aspects of the work in ensuring that questions related to the accuracy or integrity of any part of the work are appropriately investigated and resolved. Animals and procedures used in this study were approved by Animal Care and Use Committee at Shanghai Children's Medical Center, Shanghai Jiao Tong University School of Medicine (Shanghai, China). The document ID is SCMCIACUC-K2019025.

Open Access Statement: This is an Open Access article distributed in accordance with the Creative Commons Attribution-NonCommercial-NoDerivs 4.0 International License (CC BY-NC-ND 4.0), which permits the noncommercial replication and distribution of the article with the strict proviso that no changes or edits are made and the original work is properly cited (including links to both the formal publication through the relevant DOI and the license). See: https://creativecommons.org/licenses/by-nc-nd/4.0/.

\section{References}

1. Buckley JC, Heyns C, Gilling P, et al. SIU/ICUD Consultation on Urethral Strictures: Dilation, internal urethrotomy, and stenting of male anterior urethral strictures. Urology 2014;83:S18-22.

2. Chapple C, Andrich D, Atala A, et al. SIU/ICUD Consultation on Urethral Strictures: The management of anterior urethral stricture disease using substitution urethroplasty. Urology 2014;83:S31-47.

3. Fossati N, Barbagli G, Larcher A, et al. The Surgical Learning Curve for One-stage Anterior Urethroplasty: A Prospective Single-surgeon Study. Eur Urol 2016;69:686-90.

4. Frankel JK, Murphy GP. International volunteerism and urethral stricture disease: a review. Transl Androl Urol 2018;7:659-65.

5. Tian Y, Wazir R, Yue X, et al. Prevention of stricture recurrence following urethral endoscopic management: what do we have? J Endourol 2014;28:502-8.

6. Ayyildiz A, Nuhoglu B, Gülerkaya B, et al. Effect of intraurethral Mitomycin-C on healing and fibrosis in rats with experimentally induced urethral stricture. Int J Urol 2004;11:1122-6.

7. Paduszyński P, Aleksander-Konert E, Zajdel A, et al. 
Changes in expression of cartilaginous genes during chondrogenesis of Wharton's jelly mesenchymal stem cells on three-dimensional biodegradable poly(L-lactide-coglycolide) scaffolds. Cell Mol Biol Lett 2016;21:14.

8. Khalid RS, Khan I, Zaidi MB, et al. IL-7 overexpression enhances therapeutic potential of rat bone marrow mesenchymal stem cells for diabetic wounds. Wound Repair Regen 2019;27:235-48.

9. Tsai MJ, Liou DY, Lin YR, et al. Attenuating Spinal Cord Injury by Conditioned Medium from Bone Marrow Mesenchymal Stem Cells. J Clin Med 2018;8:23.

10. Liu D, Kong F, Yuan Y, et al. Decorin-Modified Umbilical Cord Mesenchymal Stem Cells (MSCs) Attenuate Radiation-Induced Lung Injuries via Regulating Inflammation, Fibrotic Factors, and Immune Responses. Int J Radiat Oncol Biol Phys 2018;101:945-56.

11. Zhao L, Liu X, Zhang Y, et al. Enhanced cell survival and paracrine effects of mesenchymal stem cells overexpressing hepatocyte growth factor promote cardioprotection in myocardial infarction. Exp Cell Res 2016;344:30-9.

12. Feng Z, Chen H, Fu T, et al. miR-21 modification enhances the performance of adipose tissue-derived mesenchymal stem cells for counteracting urethral stricture formation. J Cell Mol Med 2018;22:5607-16.

13. Liu Y, Lin L, Zou R, et al. MSC-derived exosomes promote proliferation and inhibit apoptosis of chondrocytes via lncRNA-KLF3-AS1/miR-206/GIT1 axis in osteoarthritis. Cell Cycle 2018;17:2411-22.

14. Ju C, Li Y, Shen Y, et al. Transplantation of Cardiac Mesenchymal Stem Cell-Derived Exosomes for Angiogenesis. J Cardiovasc Transl Res 2018;11:429-37.

15. Zhang X, Liu J, Yu B, et al. Effects of mesenchymal stem cells and their exosomes on the healing of large and refractory macular holes. Graefes Arch Clin Exp Ophthalmol 2018;256:2041-52.

16. Chen L, Lu FB, Chen DZ, et al. BMSCs-derived miR223-containing exosomes contribute to liver protection in experimental autoimmune hepatitis. Mol Immunol 2018;93:38-46.

17. Kornberg Z, Cooperberg MR, Spratt DE, et al. Genomic biomarkers in prostate cancer. Transl Androl Urol 2018;7:459-71.

18. Luo J, Zhao S, Wang J, et al. Bone marrow mesenchymal stem cells reduce ureteral stricture formation in a rat model via the paracrine effect of extracellular vesicles. J Cell Mol Med 2018;22:4449-59.
19. Wang LL, Yin XF, Chu XC, et al. Platelet-derived growth factor subunit $\mathrm{B}$ is required for tendon-bone healing using bone marrow-derived mesenchymal stem cells after rotator cuff repair in rats. J Cell Biochem 2018;119:8897-908.

20. Ju C, Shen Y, Ma G, et al. Transplantation of Cardiac Mesenchymal Stem Cell-Derived Exosomes Promotes Repair in Ischemic Myocardium. J Cardiovasc Transl Res 2018;11:420-8.

21. Kurt O, Yesildag E, Yazici CM, et al. Effect of Tadalafil on Prevention of Urethral Stricture After Urethral Injury: An Experimental Study. Urology 2016;91:243.e1-e6.

22. Jiang D, Gao P, Lin H, et al. Curcumin improves tendon healing in rats: a histological, biochemical, and functional evaluation. Connect Tissue Res 2016;57:20-7.

23. Ninan N, Thomas S, Grohens Y. Wound healing in urology. Adv Drug Deliv Rev 2015;82-83:93-105.

24. Barrientos S, Stojadinovic O, Golinko MS, et al. Growth factors and cytokines in wound healing. Wound Repair Regen 2008;16:585-601.

25. Liu W, Wang Y, Gong F, et al. Exosomes Derived from Bone Mesenchymal Stem Cells Repair Traumatic Spinal Cord Injury by Suppressing the Activation of A1 Neurotoxic Reactive Astrocytes. J Neurotrauma 2019;36:469-84.

26. Lou G, Chen Z, Zheng M, et al. Mesenchymal stem cellderived exosomes as a new therapeutic strategy for liver diseases. Exp Mol Med 2017;49:e346.

27. An SY, Jang YJ, Lim HJ, et al. Milk Fat Globule-EGF Factor 8, Secreted by Mesenchymal Stem Cells, Protects Against Liver Fibrosis in Mice. Gastroenterology 2017;152:1174-86.

28. Bratslavsky G, Kogan B, Levin RM. Urethra is more sensitive to ischemia than bladder: evidence from an in vitro rat study. J Urol 2001;165:2086-90.

29. Goodarzi P, Larijani B, Alavi-Moghadam S, et al. Mesenchymal Stem Cells-Derived Exosomes for Wound Regeneration. Adv Exp Med Biol 2018;1119:119-31.

30. McBride JD, Rodriguez-Menocal L, Guzman W, et al. Bone Marrow Mesenchymal Stem Cell-Derived CD63 Exosomes Transport Wnt3a Exteriorly and Enhance Dermal Fibroblast Proliferation, Migration, and Angiogenesis In Vitro. Stem Cells Dev 2017;26:1384-98.

31. Ding Q, Sun P, Zhou H, et al. Lack of endogenous parathyroid hormone delays fracture healing by inhibiting vascular endothelial growth factor-mediated angiogenesis. Int J Mol Med 2018;42:171-81. 
32. Jackson WM, Nesti LJ, Tuan RS. Mesenchymal stem cell therapy for attenuation of scar formation during wound healing. Stem Cell Res Ther 2012;3:20.

Cite this article as: Shi Z, Wang Q, Jiang D. The preventative effect of bone marrow-derived mesenchymal stem cell exosomes on urethral stricture in rats. Transl Androl Urol 2020;9(5):20712081. doi:10.21037/tau-20-833
33. Castiglione F, Dewulf K, Hakim L, et al. Adipose-derived Stem Cells Counteract Urethral Stricture Formation in Rats. Eur Urol 2016;70:1032-41. 